\title{
Identificación de gases mediante medición de complejidad
}

\author{
Mauricio Martínez M. y Miguel González-Mendoza \\ Tecnológico de Monterrey, Estado de México, México \\ A00964166@itesm.mx \\ mgonza@itesm.mx
}

\begin{abstract}
Resumen. El análisis de datos provenientes de narices electrónicas son afectados por distintos factores, cuya principal característica es la aleatoriedad. Fenómenos como flujos caóticos y concentraciones irregulares de gases o presencia de gases ajenos a un gas por detectar, dificultan a los algoritmos de clasificación empleados en esta área su identificación. Debe considerarse también que la estructura de los vectores de estos datos son de alta dimensionalidad y pocas muestras, lo que dificulta descubrir patrones en ellos. En este trabajo se empleó el concepto de Medición de Complejidad, el cual define la cantidad de desorden presente en un sistema. Para demostrar la eficiencia de este concepto en la identificación de olores, se analizó un conjunto de mediciones de una nariz electrónica expuesta a mezclas de etileno, metano y monóxido de carbono a distintas concentraciones y bajo condiciones de flujo de gases muy cercanos a la realidad. Los resultados indican que la medición de complejidad logra discriminar los gases que constituyeron las distintas mezclas, identificando el desempeño de los sensores para reconocerlos de acuerdo a las magnitudes de complejidad y desorden.
\end{abstract}

Palabras clave: Medición de complejidad, sensores MOX, identificación de gases, clasificación por medición de complejidad.

\section{Introducción}

El empleo de narices electrónicas en el reconocimiento de aromas tiene diversas aplicaciones en las áreas de la industria, medicina, monitoreo ambiental, agricultura, etc. [1]. Estos dispositivos construidos bajo distintas tecnologías basan su funcionamiento en la reactividad que ciertos materiales tienen con determinados gases y manifiestan su presencia al afectar algunas de sus propiedades como son la resistencia, capacitancia, transconductancia, piezoeléctricidad entre otras [1] [2]. El uso de tecnología de semiconductores en esta área ha permitido la construcción de narices electrónicas de bajo costo; es el caso de los dispositivos MOX (Metal Oxide Semiconductor) cuya alta sensibilidad a determinados gases, se manifiesta con variaciones de transconductancia. El análisis de este tipo de mediciones tiene como objetivo mejorar la capacidad de discriminación de gases detectados por las narices electrónicas. Este proceso se ve afectado por 
distintos factores como son la calibración del dispositivo, la forma en que se dispersan los gases, la presencia de otros gases o la concentración que tienen [2-4]. La naturaleza de los datos implica otra serie de dificultades para los algoritmos empleados en su análisis; son vectores de alta dimensionalidad y un número mínimo de los mismos. El proceso de análisis de esta información se desarrolla en las etapas de preprocesamiento, reducción y selección de atributos para finalmente llevar a cabo un proceso de clasificación o identificación del olor en el caso de la nariz electrónica [5-7].

Los investigadores en esta área están interesados en determinar el desempeño de los sensores para identificar, discriminar y conocer el grado de concentración de distintos olores en tiempo real [8-11]. Lo que permitiría detectar la fuga de combustibles, gases explosivos o tóxicos, o el monitoreo oportuno de calidad del aire entre otras situaciones donde el tiempo es crítico. Distintos algoritmos provenientes del campo de aprendizaje de máquina se emplean actualmente en este tipo de información en la reducción, selección de atributos y clasificación; esta última actividad tiene la dificultad de presentar una respuesta lenta debido al proceso de entrenamiento que requiere, y la cual debe llevarse a cabo nuevamente cuando los sensores fallan o terminan su vida útil. Además, establecer un proceso de clasificación de los datos es difícil, debido al comportamiento que tienen los sensores para su estabilización en el tiempo, y las lecturas de transconductancia presentan un cierto grado de aleatoriedad; aún trabajando en condiciones similares. Los algoritmos empleados comúnmente en estos procesos van desde K-NN (K nearest neighbors), Redes neuronales, Máquinas de Soporte Vectorial (SVM), Modelos de densidad, etc. [12-14].

El análisis de de datos obtenidos de narices electrónicas busca determinar la relación que existe entre un conjunto de variables independientes; lecturas de salida de un arreglo de $n$ sensores, y un conjunto de variables dependientes denominadas olores. Cada sensor generará una respuesta en el tiempo, asociada al olor para el que fue diseñado reconocer. Por lo tanto, la salida de un arreglo de sensores o nariz electrónica tendrá una representación $X_{i j}(t)$, donde $i$ representa algún sensor de la nariz y $j$ el olor que reconocen [15].

Lo anterior representa un problema de clasificación, los algoritmos empleados en ésta área están basados en técnicas estadísticas o paramétricas; los cuales emplean las características de las distribuciones de datos como la media y desviación estándar para establecer la relación entre las salidas de los sensores y los olores que reconocen o clases. En tanto que los no paramétricos son implementados mediante técnicas de regresión lineal, rangos, distancia, etc. de tal forma que de las lecturas de los sensores, se pueda discernir su pertenencia a una instancia o clase. La forma en que se realizan las actividades anteriores pueden ser de forma supervisada, y esto se refiere a que durante el proceso de clasificación existe un conocimiento previo de relación entre datos y clases. El cual es utilizado para determinar la pertenencia de nuevos vectores de datos a estas clases mediante un proceso de aprendizaje o entrenamiento ya sea por calibración, correlación, regresión, etc. En cambio, la clasificación no supervisada no dispone de un conocimiento previo y separa los vectores de datos mediante 
técnicas de agrupación, proyección lineal, etc. $[2,15]$.

Los algoritmos de clasificación al enfrentar la aleatoriedad tienen problemas en la identificación de regularidades durante el proceso de clasificación; algunos de ellos, demandan un conjunto más grande de muestras para mejorar su desempeño. Por otra parte, La naturaleza de los datos provenientes de narices electrónicas es de alta dimensionalidad y un número de muestras mínimo; lo que implica una demanda computacional alta, durante el proceso de entrenamiento de los algoritmos de clasificación [16]. Además, el proceso de aprendizaje tiene que ser llevado a cabo nuevamente cuando la nariz electrónica es ajustada y alguno o varios de sus sensores son reemplazados por deterioro.

Considerar la aleatoriedad como una característica de análisis en lecturas de salida de narices electrónicas ha sido pocas veces abordada. La medición de esta característica fue planteada por C. Shannon en su concepto de Entropía de información y desde entonces se han derivado varias técnicas de análisis de datos basados en él; ecuación 1 [17]. Se pueden mencionar Ganancia de Información, Máxima Entropía, Divergencia de Kullback-Leibler, entre otros. Estos algoritmos están clasificados entre las técnicas de selección y reducción de atributos. Basan su funcionamiento en la evaluación de la entropía de los datos y la valoración de su capacidad de ser informativos bajo este concepto. Son rápidos y permiten superar con facilidad la alta dimensionalidad que presentan algunos conjuntos de datos. La aleatoriedad es una característica que puede ser medida en ellos, y todo elemento aporta la información pertinente sin ser sesgado por la influencia de otros datos.

Entre los investigadores que han empleado conceptos derivados de entropía de información en la clasificación de olores con narices electrónicas están Alexander Vergara et al. [18] quienes utilizaron la distancia de Kullback-Leibler para identificar gases, calculando la divergencia de información que existe entre las distintas distribuciones de probabilidad de datos obtenidos de las lecturas de una nariz electrónica. Entendiendo por divergencia de información el grado de discrepancia que existen entre un par de distribuciones medido por el grado de entropía de de información mutua que poseen. El experimento llevado a cabo por Alexander Vergara tuvo como objetivo distinguir dos mezclas de gases; una de etanol con acetaldehído y acetona, otra de etanol con etileno. Los autores afirman que tuvieron una eficiencia del 100 por ciento en la discriminación de los gases analizados bajo condiciones controladas y liberación directa de los gases sobre los sensores. Otro trabajo que reporta el uso de teoría de la información en el análisis de datos provenientes de narices electrónicas lo llevaron a cabo X. Rosalind Wang et al. [6] empleando máxima información mutua. Este concepto es una variante de Kullback-Leibler que determina la similaridad de dos distribuciones de datos, calculando la entropía de su probabilidad conjunta y el producto de sus marginales. Con lo anterior los autores fueron capaces de descubrir los atributos más informativos contenidos en los datos que colectaron y los utilizaron en el proceso de clasificación de los gases mediante Máquinas de Soporte Vectorial y t-test. Concluyeron que los algoritmos SVM incrementan su eficiencia y para el caso de t-test no existe una mejora notable. 
El objetivo de este trabajo es aplicar el concepto de medición de complejidad en el proceso de identificación de gases mediante sensores MOX. Demostrando, que un algoritmo de clasificación basado en este concepto, identifica de manera rápida y clara los gases sensados, e identificando el grado de aleatoriedad que tienen los datos ante las circunstancias fortuitas bajo las que se realiza el proceso de colecta de datos. Jordi Fonollosa et al. desarrollaron un experimento de detección de gases en condiciones muy similares a las condiciones de trabajo en las que son utilizadas las narices electrónicas; pusieron a disposición sus conjuntos de datos y se evaluará el desempeño del algoritmo propuesto en este trabajo con los datos de Jordi Fonollosa [19].

\section{Métodos}

El concepto de Medición de complejidad ha sido planteado por J. S. Shiner y Matt Davison como una función del desorden u orden que posee un sistema, ecuación 5. A su vez, el desorden es expresado como la entropía normalizada de los datos con respecto al nivel máximo de entropía que estos tendrían bajo una distribución uniforme, ecuaciones 3 y 2 respectivamente [20].

$$
\begin{aligned}
S & =\sum_{i=1}^{n}-P_{i} \log _{2} P_{i} \\
S_{\text {max }} & =\log _{2} N \\
\Delta & \equiv S / S_{\text {max }} \\
\Omega & \equiv 1-\Delta \\
\Gamma_{\alpha \beta} & \equiv \Delta^{\alpha} \Omega^{\beta}
\end{aligned}
$$

La aplicación de este concepto en datos muestreados en series de tiempo es simple, ya que las mediciones muestran eventos identificables y es posible determinar su frecuencia a partir de las lecturas obtenidas de los sensores MOX. El concepto de entropía de información se basa en la identificación de la probabilidad de eventos que conforman el conjunto de datos de algún experimento y la valoración de estos por su capacidad de ser informativos, ecuación 1.

La forma de aplicar el concepto de Medición de complejidad se hace de la manera más simple; los parámetros $\alpha$ y $\beta$ se establecen con valor uno y produce la expresión más sencilla de medición de complejidad; la cuadrática.

Las condiciones del experimento llevado a cabo por Jordi Fonollosa et al. [19] en la detección de mezclas de Etileno con Monóxido de Carbono (CO) o Metano a distintos concentraciones son reportadas en la sección de materiales; donde se describe la forma en que se realizaron las mezclas y su dispersión que simuló turbulencias reales.

\subsection{Materiales}

El experimento utilizó un túnel de viento con un volumen de cámara de $2.5 \times 1.2 \times 0.4 \mathrm{~m}^{3}, 3$ fuentes de gas de flujo controlado conteniendo etileno, metano 
y monóxido de carbono respectivamente, y un arreglo de 8 sensores MOX. Los sensores detectan cuatro gases: Etileno, Metano, monóxido de Carbono (CO) y Propano; Tabla 1.

Tabla 1. Sensores MOX con material que sensan [19]

\begin{tabular}{ccc}
\hline \hline Tipo de sensor Número de unidades & Gas que detectan \\
\hline TGS2600 & 1 & Hidrógeno, Monóxido de carbono \\
TGS2602 & 2 & Amonio, Compuestos Organicos Volátiles (VOC) \\
TGS2610 & 1 & Petano \\
TGS2611 & 1 & Metano, Propano, Butano \\
TGS2612 & 1 & Monóxido de carbono, Gases de combustible, VOC \\
TGS2620 & 2 &
\end{tabular}

\subsection{Protocolo}

Se propusieron treinta y cuatro experimentos con seis mediciones cada uno. Cada experimento es la combinación de distintas concentraciones de los gases a sensar, etiquetados en cuatro niveles: cero, bajo, mediano y alto. El flujo de los gases se realizó en condiciones estándar a $0^{\circ} \mathrm{C}$ y un atm y su concentración fue manejada en partes por millón (ppm); Tabla 2.

Tabla 2. Tabla de experimentos [19]

\begin{tabular}{|c|c|c|c|c|c|}
\hline \multicolumn{6}{|c|}{ Etileno @ 2500 ppm } \\
\hline & & \multicolumn{4}{|c|}{$20 \mathrm{sccm} 14 \mathrm{sccm} 8 \mathrm{sccm} 0 \mathrm{sccm}$} \\
\hline \multirow{4}{*}{ Metano@ 1000 ppm } & $300 \mathrm{sccm}$ & 6 & 6 & 6 & 6 \\
\hline & $200 \mathrm{sccm}$ & 6 & 6 & 6 & 6 \\
\hline & $100 \mathrm{sccm}$ & 6 & 6 & 6 & 6 \\
\hline & $0 \mathrm{sccm}$ & 6 & 6 & 6 & \\
\hline \multirow{4}{*}{ CO@ 9000 ppm } & $200 \mathrm{sccm}$ & 6 & 6 & 6 & $\overline{6}$ \\
\hline & $140 \mathrm{sccm}$ & 6 & 6 & 6 & 6 \\
\hline & $80 \mathrm{sccm}$ & 6 & 6 & 6 & 6 \\
\hline & $0 \mathrm{sccm}$ & 6 & 6 & 6 & \\
\hline
\end{tabular}

Las salidas de medición de los sensores (transductancia) fueron registradas durante $300 \mathrm{~s}$ de acuerdo al siguiente esquema: $60 \mathrm{~s}$ sin la liberación de ningún gas, en el segundo 60 las fuentes de gas son abiertas de acuerdo a la combinación de gases a experimentar y la razón de flujo especificada (cero, baja, mediana y alta), esto se hace durante $180 \mathrm{~s}$; después de este tiempo se cierra el flujo de 
gas en las fuentes y se esperan $60 \mathrm{~s}$ hasta alcanzar la recuperación de línea base de los sensores. En cada experimento la señal de los sensores fue muestreada cada $20 \times 10^{-3} \mathrm{~s}$; reportando también la humedad relativa y la temperatura. lo anterior genera 8 vectores de mediciones correspondientes al número de sensores empleados en la nariz electrónica.

\subsection{Algoritmo}

Los vectores de medición de transconductancia de cada sensor, fueron normalizados bajo el concepto de Escalamiento de atributos, esto permite asegurar la comparación entre los vectores compensando las diferencias de escalas entre los mismos; pues coloca los datos entre un rango de cero y uno.

Al tener identificados los diferentes experimentos se dispone de un conjunto de clases que pueden ser distinguidas entre sí (Tabla 2). Cada experimento corresponde a una clase; y ésta a su vez, corresponde a una mezcla específica de gases. Por cada clase, se elige aleatoriamente un conjunto de datos, con los que se calcula el desorden de la transconductancia normalizada de las lecturas obtenidas por cada sensor; para posteriormente obtener su medición de complejidad. Mediante un gráfico de Medición de complejidad contra desorden se ilustra el desempeño que cada sensor tiene para detectar el gas para el que fue diseñado en función de las etiquetas de cero, bajo, mediano y alto. El algoritmo para realizar lo anterior se presenta en el procedimiento Complejidad.

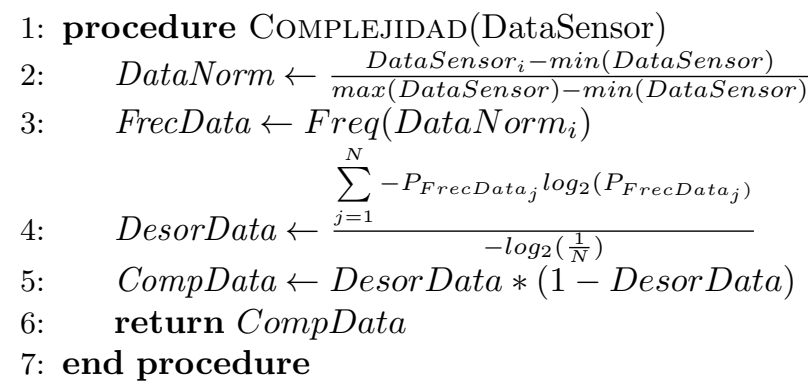

\section{Resultados}

La presentación de los resultados se organizó de acuerdo al tipo de gas que detectan los sensores y estos fueron agrupados para comparar el desempeño que cada uno de ellos tiene en cada experimento. Los gráficos de medición de complejidad contra desorden de los sensores TGS2602 y TGS2602.1 fueron anexos entre sí por que sensan etileno (gas base), los sensores TGS2611 y TGS2612 por que sensaron metano, TGS2600, TGS2620 y TGS2620.1 monóxido de carbono y el sensor TGS2610 propano. Por ejemplo; en el experimento de etileno en cualquiera de sus concentraciones mezclado con monóxido de carbono a nivel medio mostró el desempeño del algoritmo y la capacidad de los sensores TGS2602, TGS2602.1 y TGS2600, TGS2620 y TGS2620.1 para identificar Etileno y CO respectivemente; 
conforme se aumenta la cantidad de etileno, la complejidad de los datos de los sensores que lo sensan también lo hacen. Lo anterior supone una reducción del desorden; Fig. 1.
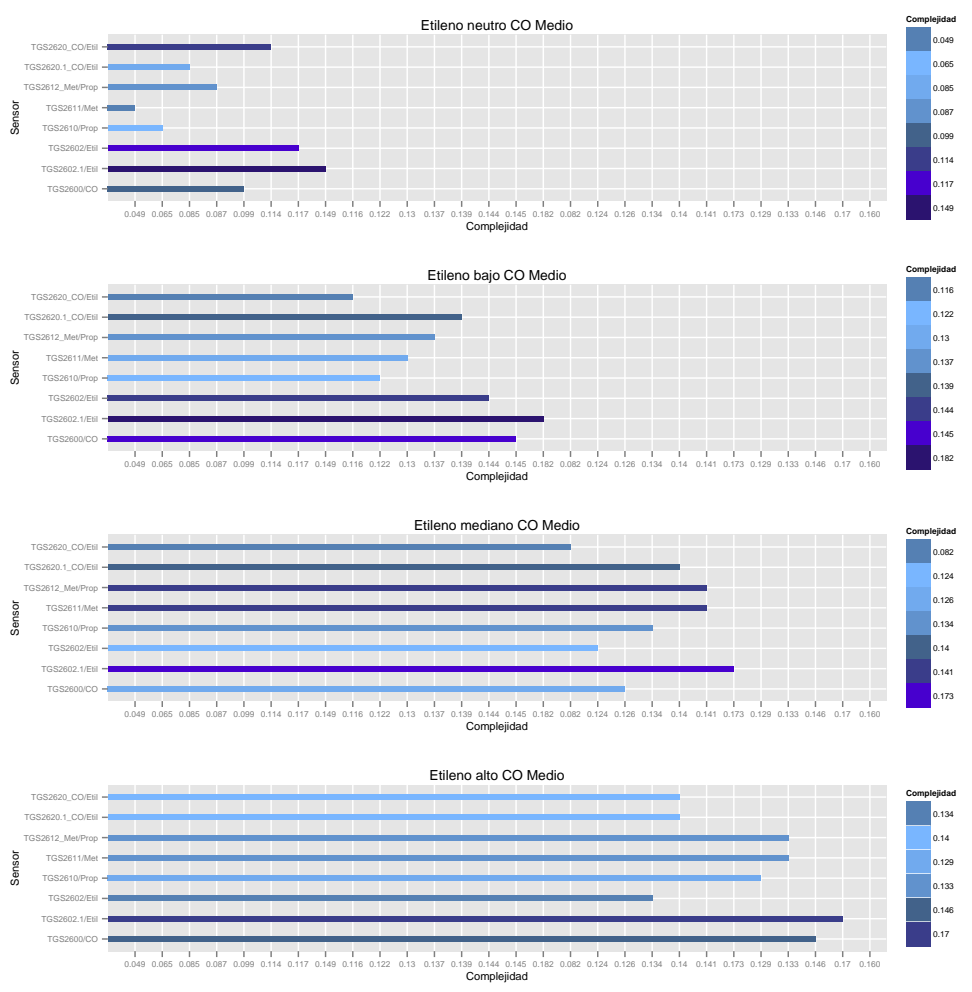

Fig. 1. Medición de complejidad por sensor. Experimento Etileno con CO medio

El arreglo de gráficas representa cada uno los sensores que constituyen a la nariz electrónica utilizada en el experimento. Cada gráfica muestra la Medición de complejidad correspondiente a las medidas de transconductancia de cada sensor. La variaciones de concentración de los gases mezclados se reflejan en cambios de magnitud de complejidad.

La complejidad en sensores como TGS2600, TGS2620 y TGS2620.1; los cuales detectan CO para el primero y VOC y CO para los dos últimos, muestran un aumento de complejidad conforme aumenta la concentración de etileno. Solo considérese que el etileno está catalogado como un VOC ( Compuesto Orgánico Volátil ); Fig. 1.

En los experimentos de etileno en sus distintas concentraciones con CO y Metano altos presentaron un comportamiento en complejidad similar al de 
etileno con CO y Metano medio; Fig. 2. Sin embargo, el caso de etileno con CO y Metano bajos los sensores específicos para estos dos gases mostraron su más alto nivel del complejidad en ausencia de etileno o en un nivel bajo del mismo.

Finalmente, la detección de etileno en sus distintas concentraciones sin mezcla de CO o metano permitió observar un aumento natural de la complejidad en las mediciones de transconductancia de todos aquellos sensores que detectan este gas y una complejidad baja para el sensor de CO; excepto el sensor para propano; TGS2610, el cual presentó un aumento en la complejidad de sus mediciones para cada aumento de concentración de etileno.
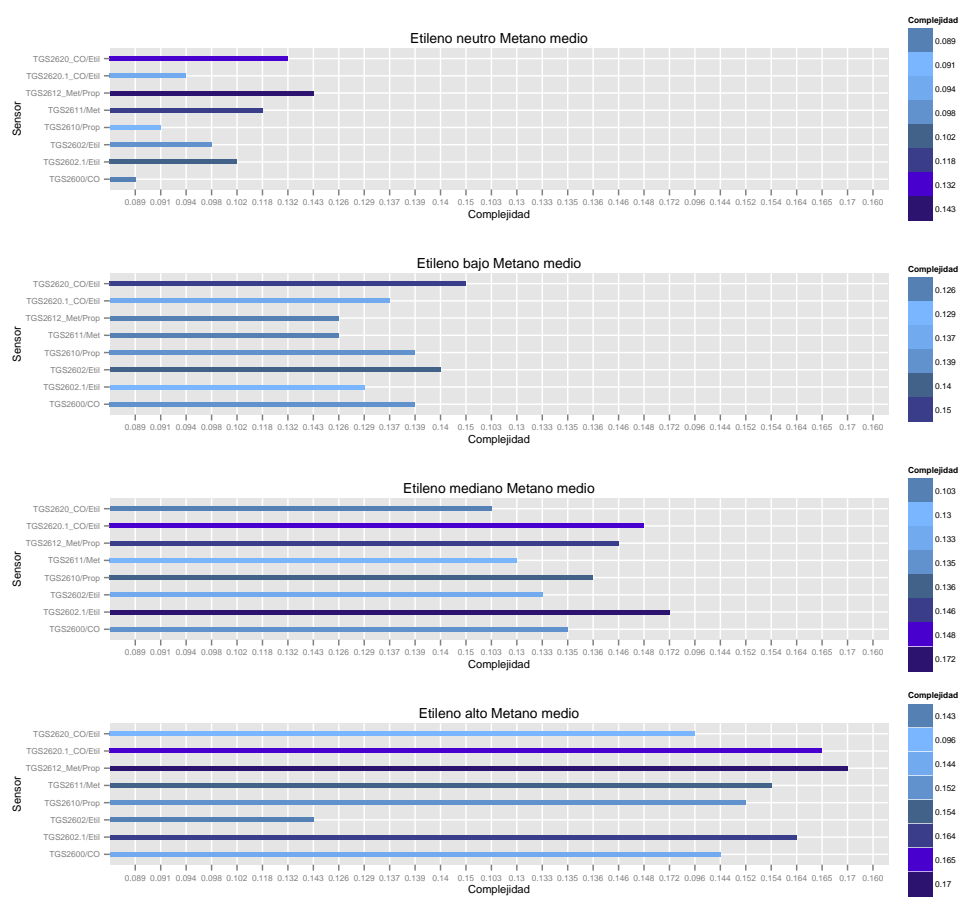

Fig. 2. Medición de complejidad por sensor. Experimento Etileno con Metano medio

\section{Discusión}

Los trabajos de Jordie Fonollosa et al. y Sepideh Pashami et al. [19], [14] plantean la identificación de gases en ambientes turbulentos y la detección del cambio de sus concentraciones como las condiciones reales bajo las cuales trabajan las narices electrónicas. Consideran oportuno tomar en cuenta lo anterior principalmente en aplicaciones donde el tiempo es un factor crítico; como en 
la fuga y detección de gases tóxicos. Aunque emplearon Máquinas de Soporte Vectorial Inhibitorias (ISVM) y Razón de Verosimilitud generalizada (GLR); una variante del cálculo de verosimilitud, estos no dejan de ser afectados por la incertidumbre de los datos y la alta dimensionalidad de las muestras.

La medición de complejidad en este aspecto resulta ventajosa dadas las características que aporta el concepto base del mismo; que es la entropía de información. Ante un evento seguro (lecturas de mediciones constantes) o la ausencia de eventos, la entropía es cero; Eventos de baja probabilidad determinan bajos niveles de entropía y una distribución uniforme de probabilidad de los mismos supone el máximo valor de entropía que un sistema puede tener.

Ahora bien, existe una reducción de dimensiones al mapear las distintas lecturas de un sensor, al contar por el número de ocurrencias con el que pueden ser representadas bajo un conjunto finito de ellas o eventos. La forma de la distribución de los datos no afecta el análisis bajo este concepto; como lo pueden ser los métodos de estadísticos. La interpretabilidad es otra ventaja; si los datos presentan un alto de nivel de entropía no se puede aseverar una tendencia entre ellos por su misma aleatoriedad; pero si esta es baja, comienza entonces a reconocerse cierta predictibilidad en los datos y por tanto la detección de un patrón o clase.

Jordie Fonollosa et al. reportan una precisión de clasificación del 97 por ciento en la clasificación de las distintas mezclas de gases que analizaron empleando ISVM. En el mismo artículo muestran el comportamiento de las mediciones de transconductancia de los sensores que componen la nariz electrónica, durante los 300 s de cada prueba. De los cuales; solo dos sensores despliegan un comportamiento discriminativo en sus señales, los cuales es de suponer son los de compuestos orgánicos volátiles a los cuales pertenece el etileno. En tanto que el resto de las señales se traslapan en cierto grado y pertenecen al resto de los sensores que no se especializan en él. Si Jordie Fonollosa et al. evalúan en forma global la detección de etileno con las lecturas de los distintos vectores es posible que la mayoría afectará la capacidad de clasificación de la ISVM utilizada; pues solo dos sensores estaban dedicados al etileno; además de tener que superar los factores ya descritos.

En el caso de medición de complejidad; el análisis fue por sensor, y los resultados muestran la magnitud de esta característica que cada conjunto de mediciones de transconductancia sustenta. Bajo las circunstancias en que se desarrollaron los experimentos, los gráficos de complejidad muestran la capacidad de cada sensor para identificar los componentes de las mezclas de gases o hacer evidente la incapacidad de los sensores para identificarlos.

Es de notar que la capacidad de clasificación o discriminación de los sensores mediante los conceptos de medición de complejidad y desorden permiten interpretar el desempeño de los mismos. Magnitudes bajas de complejidad y de alto desorden, implica que las lecturas de transconductancia no definan una tendencia clara o lo que es lo mismo; la distribución de probabilidad de sus mediciones tiende a ser uniforme. En el análisis por medición de complejidad no existen respuestas determinísticas, pues se enfrenta a un fenómeno afectado por 
factores no controlables, por tanto es más natural presentar la clasificación de sus resultados en función de una medida de aleatoriedad. El algoritmo no necesita de entrenamiento, puede ser clasificado dentro de los métodos no supervisados como un método de rangos. Permite distinguir puntos de cambio en la concentración de los gases y la separación de los estatus (clases) o su falta de ella.

\section{Conclusiones}

La aplicación del concepto de medición de complejidad en la identificación y clasificación de gases es propiamente un reflejo de comportamiento transitivo que tienen las lecturas de transconductancia en los sensores ante los cambios que se presentan en la detección de gases. Este método es rápido e independiente de la distribución estadística de los datos por lo tanto no depende de la elección de un modelo para el proceso de clasificación. En el aspecto de alta dimensionalidad existe una reducción de esta característica al mapear los datos a un conjunto de eventos finitos junto con sus frecuecias. Puede visualizarse el grado de separación o traslapamiento de las clases a identificar, pero no ofrece una respuesta definitiva de clasificación.

\section{Referencias}

1. K. Arshak, E. Moore, et al.: A review of gas sensors employed in electronic nose applications. Emerald Group Publishing. Sensor Review (2004)

2. Leonardo Tomaseli Duarte: Design of Smart Chemical Sensor Array: an Approach Based on Source Separation Methods. Institut Polytechnique de Grenoble (2010)

3. Javier G. Monroy, Javier González-Jiménez, Jose Luis Blanco: Overcoming the Slow Recovery of MOX Gas Sensors through a System Modeling Approach. Open Access Sensors (2012)

4. Kok Seng Eu, Kian Meng Yap: Overcoming Long Recovery Time of Metal-Oxide Gas Sensor with Certain Factor Sensing Algorithm. In: Proceedings of the 8th International Conference on Sensing Technology (2014)

5. Ayoub Einollahi: Selectivity Enhancement for Temperature Modulated Electronic Nose Using Phase Space and Dynamic Moments. Orebro University (2012)

6. X. Rosalind Wang, Joseph T. Lizier et al.: Feature Selection for Chemical Sensor Arrays Using Mutual Information. PLOS one Open Acces (2014)

7. Thomas Nowotny, Amalia Z. Berna, et al.: Feature selection in Enose applications. PLOS one Open Acces (2014)

8. V. E. Bochenkov, G. B. Sergeev: Sensitivity, Selectivity, and Stability of GasSensitive Metal-Oxide Nanostructures. American Scientific Publishers (2010)

9. Javier G. Monroy, Achim Lllienthal, et al.: Calibration of MOX gas sensors in open sampling systems based on Gaussian Processes. (2011)

10. Hasim Alam, S. Hasan Saeed: Subharmonic solutions with prescribed minimal Modern Applications of Electronic Nose: A review. International Journnal of Electricall Computer Enginnering (2013)

11. X. Rosalind Wang, Joseph T. Lizier et al.: Human breath-print identification by E-nose, using information-theoretic feature selection prior to classification. Sensors and Actuators B: Chemical, Elsevier (2014) 
12. Amine Bermak, Sofiane Brahim Belhouari, et al.: Patttern Recognition Techniques for Odor Discrimination in Gas Sensor Array. American Scientific Publishers (2006)

13. Ricardo Gutierrez-Osuna: Pattern Analysis for Machine Olfaction: A review. IEEE Sensors Journal (2002)

14. Sepideh Pashami, Achim J. Lllienthal, Marco Trincavelli: Detecting Changes of a Distant Gas with an Array of MOX Gas. Open Access Sensors (2012)

15. Evor L. Hines, et al.: Pattern Analysis for Electronic Noses. Handbook of Machine Olfaction: Electronic Nose Technology. Edited by T.C. Pearce, S.S. Schiffman, H.T. Nagle, J.W. Gardner (2003)

16. Martin Lngkvist, Lars Karlsson, Amy Loutfy: A review of unsupervised feature learning and deep learning for time-series modeling. Pattern Recognition Letters, Elsevier (2013)

17. C. E. Shannon: A Mathematical Theory of Communication. The Bell System Technical Journal, Vol. 27, pp. 379-423, 623-656 (1948)

18. Alexander Vergara, Mehmet K. et al.: Kullback-Leibler distance optimization for artificial chemo-sensors. IEEE Sensors Journal (2009)

19. Jordi Fonollosa, Irene Rodríguez-Lujan et al:: Chemical Discriminatio in Turbulence Gas Mixtures with MOX Sensors Validated by Gas Chromotography-Mass Spectrometry. Open Access Sensors (2014)

20. J. S. Shiner, Matt Davison: Simple measure for complexity. The American Physical Society, Physical Review, Volume 59, number 2 (1999) 